\title{
Cefdinir-Associated "Bloody Stools" in an Infant
}

\author{
Reese Graves, MD, and Sally P. Weaver, PhD, MD
}

Cefdinir, a third-generation oral cephalosporin frequently used in pediatric populations, may cause red stools when administered with iron or products that contain iron, such as infant formula. This benign side effect is not well documented in the medical literature. We describe a 7-month-old girl who was evaluated for red stools while taking cefdinir along with an oral iron supplement. (J Am Board Fam Med 2008;21:246-248.)

Cefdinir is a commonly prescribed antibiotic in pediatric populations because of its once-daily dosing and its indications for acute otitis media, pharyngitis, acute sinusitis, and uncomplicated skin infections. Maroon- or red-colored stools are a known side-effect of cefdinir that have been infrequently reported in the medical literature. The manufacturer states that "there have been reports of reddish stools in patients receiving cefdinir," and adds that in many of these reports, patients were also receiving products containing iron. ${ }^{1}$ According to the manufacturer, cefdinir or its metabolites combine with iron and form a precipitate which gives a characteristic discoloration to stool. The complex has no known adverse effects and stools return to normal color after discontinuing cefdinir.

\section{Case Report}

GR, a 7-month-old girl born at 31 weeks' gestation, had a history of apnea of prematurity and dietary anemia and who presented to a community emergency department with the complaint of maroon-colored stools. Nine days before her presentation, GR was evaluated by her primary physician for 2 episodes of blood-streaked stools and constipation. At that time, her stool was examined and found to be guaiac positive. Examination revealed 2

This article was externally peer reviewed.

Submitted 30 October 2007; revised 4 February 2008; accepted 7 February 2008.

From the McLennan County Medical Education and Research Foundation, Waco, Texas.

Funding: none.

Conflict of interest: none declared.

Corresponding author: Sally Weaver, MD, McLennan Co. Medical Education and Research Foundation, Family Health Center, 1600 Providence Drive, Waco, TX 76707 (E-mail: spweaver@juno.com). small anal fissures. Because of concerns that the hematochezia was more than could be attributed to anal fissures, hemoglobin levels, abdominal plain films, and a Meckel's scan were obtained. She was started on polyethylene glycol 3350-oral for constipation and topical diaper rash ointment for the anal fissures and continued on her regular supplemental iron drops. Her hemoglobin was 11.5 and both the abdominal plain films and Meckel's scan were negative. According to GR's mother, reevaluation 2 days later revealed no further blood in her stools. Six days before presentation, GR began a course of cefdinir $100 \mathrm{mg}$ once daily by mouth for recurrent acute otitis media.

At the emergency department, GR's mother reported that GR had 2 voluminous red stools. She reported that her daughter was otherwise normal, taking her usual amount of formula, having soft stools, and not particularly fussy. A dietary history was performed and GR's mother denied giving foods with prominent dyes. During examination, GR was afebrile with normal vital signs and was alert and playful. A gastrointestinal examination revealed normal, active bowel sounds; a nontender, nondistended abdomen; a small anal fissure; and no rashes during a skin examination. GR's diaper contained red-colored soft stool that was guaiac negative on 2 separate tests performed in the emergency department. Blood laboratory tests revealed hemoglobin level of 11.9 and white blood cell count, platelet count, coagulation studies, and comprehensive metabolic panel all within normal limits. Because of concerns that the guaiac tests were falsenegatives, GR was admitted to the pediatric hospital service for observation. Over the next several hours she remained clinically stable but continued to have red stools. After careful review of her med- 
ication list, which included ferrous gluconate 12.5 $\mathrm{mg}$ daily, cefdinir $100 \mathrm{mg}$ daily, polyethylene glycol $33505 \mathrm{~g}$ daily, and cimetidine $150 \mathrm{mg}$ daily, the hospital team concluded that the discolored stools were attributable to a medication interaction. Using the Naranjo Nomogram, the likelihood that GR's stool discoloration was caused by cefdinir was estimated as probable. ${ }^{2}$ GR's mother was informed of the interaction between cefdinir and iron and reassured that the discoloration of the stool would resolve after completing the cefdinir. At follow-up and no longer taking cefdinir, GR had had no further episodes of red stools.

\section{Literature Review}

Red stools from cefdinir are described in the package insert ${ }^{1}$ but only 3 cases are reported in the medical literature. ${ }^{3-5}$ A search of Medline, 1996 to present, using the MESH search terms "cefdinir," "adverse effects," and/or "drug/food interactions" and search terms "red stool" or "maroon stool" revealed only 1 published case report of red stools caused by cefdinir. ${ }^{3}$ This was a short case report of 2 subjects published as a letter to the editor. ${ }^{3}$ The 2 patients reportedly developed "brick-red" stools while simultaneously taking cefdinir and iron-fortified formula.

There were no reports of stool discoloration caysed by cefdinir in the Database of Abstracts of Reviews of Effects or in TOXNET. A search of www.scholar.google.com for "red stool" and "cefdinir" revealed 2 additional reports of red stools related to cefdinir use. One was merely a republishing of the aforementioned case report ${ }^{4}$ and the other an anecdotal report of red stools related to cefdinir use in the child of an academic pediatrician. ${ }^{5}$ Finally, a search of www.google.com for "red stools" and "cefdinir" resulted in many parental reports of cefdinir causing either red- or maroon-colored stools in their children, or medication web sites reporting stool color change as a possible side effect of cefdinir.

\section{Discussion}

The incidence of red stools with cefdinir is unknown. One small study of cefdinir tolerability in children incidentally reported the side effect of the development of red discoloration in stool occurred in nearly $10 \%$ (4 of 39) of children treated with cefdinir. ${ }^{6}$ The Sanford Guide reports a $1 \%$ incidence of red stools when using cefdinir. ${ }^{7}$ Iron sup- plements are known to reduce cefdinir absorption by $31 \%$ to $80 \%$, although formula containing iron does not affect absorption. ${ }^{1}$ The amount of iron coadministered with cefdinir necessary to create red stools is unknown; however, the amount needed to form the iron cefdinir complex is probably very low based on the case reports of red stools forming while taking formula that contains iron. ${ }^{3}$

Despite the benign nature of the cefdinir iron complex, red stools can create distress and confusion among both parents and physicians. In the above case, extra laboratory testing was obtained and the patient was admitted to the hospital overnight for a benign medication side effect. Greater awareness of this medication side effect allows for the education of patients before the drug is prescribed and the reassurance of patients concerned by the alarming appearance of red stools. Presumably, adherence to medication may also be improved in those experiencing this side effect if patients are educated in advance that this stool color change may occur. Among physicians, familiarity with this side effect is somewhat limited. Physicians familiar with the characteristic red stools with cefdinir use will more efficiently arrive at the correct diagnosis of a drug interaction, sparing both time and resources that might otherwise go into further investigation of the discolored stools.

\section{Conclusion}

Physicians regularly prescribing cefdinir should be aware that red stools are a possible side effect. Although this side effect has only been reported in children, it is also a possibility in adults taking cefdinir along with products containing iron. This side effect can be induced in children who ingest relatively small amounts of iron, such as that found in infant formula. The evaluation of a patient taking cefdinir and presenting with red stools can be limited to a stool guaiac test or no testing at all. Red stools are not an indication for discontinuing cefdinir and should resolve once the course of cefdinir is completed. Awareness of this side effect should eliminate unnecessary hospitalizations and testing, decrease nonadherence, and assuage parental anxiety.

\section{References}

1. Omnicef package insert. Chicago, IL: Abbott Laboratories. July 2005.

2. Naranjo CA, Busto J, Sellers EM, et al. A method for 
estimating the probability of adverse drug reactions. Clin Pharmacol Ther 1981;30:239-45.

3. Nelson JS. Red stools and Omnicef. J Pediatr 2000; 136:853-4.

4. Cefdinir: red stools in a child and an infant: 2 case reports. Reactions 2000;1:6.

5. Nelson JD, McCracken GH. Red stools and Omnicef. Ped Infect Dis J 2002;21:A7-A8.
6. Bowlware KL, McCracken GH, Lozano-Hernandez J, Ghaffar F. Cefdinir pharmacokinetics and tolerability in children receiving $25 \mathrm{mg} / \mathrm{kg}$ once daily. Ped Infect Dis J 2006;25:208-10.

7. Gilbert DN, Moellering RC, Eliopoulos GM, Sande MA. The Sanford Guide to Antimicrobial Therapy 2007. 37th edition. Sperryville (VA): Antimicrobial Therapy, Inc.; 2007:87. 